\title{
Fitting strategies to extract the axial charge of the nucleon from lattice QCD
}

\author{
Georg M. von Hippel ${ }^{1}$, Jiayu Hua*1, Benjamin Jäger ${ }^{1,2}$, Harvey B. Meyer ${ }^{1,2}$, \\ Thomas D. Rae ${ }^{1}$, Hartmut Wittig ${ }^{1,2}$ \\ ${ }^{1}$ PRISMA Cluster of Excellence and Institut für Kernphysik, University of Mainz, \\ Johann-Joachim-Becher-Weg 45, 55099 Mainz, Germany \\ ${ }^{2}$ Helmholtz Institut Mainz, University of Mainz, 55099 Mainz, Germany \\ E-mail: hua@kph.uni-mainz.de
}

We report on a comparison of several fit methods used for the extraction of the nucleon axial charge $g_{\mathrm{A}}$ from lattice QCD with two dynamical flavours of $\mathrm{O}(a)$ improved Wilson quarks. We use plateau fits, summed operator insertions (the summation method) and a new "midpoint" method to investigate contributions from excited states that affect the determination of $g_{\mathrm{A}}$. We also present a method to perform correlated fits when the standard estimator for the inverse of the covariance matrix becomes unstable.

31st International Symposium on Lattice Field Theory LATTICE 2013

July 29 - August 3, 2013

Mainz, Germany

${ }^{*}$ Speaker. 


\section{Introduction}

Lattice QCD allows for the determination of nucleon structure quantities such as the axial charge, $g_{\mathrm{A}}$, which is well known experimentally from neutron beta decay and which can be determined on the lattice without any extrapolation in the momentum transfer, making it an important benchmark quantity for lattice QCD. For an accurate determination of $g_{\mathrm{A}}$, it is important to take excited-state contributions into account. In this context, a particular difficulty arises when fully correlated fits become unstable; here, we explore possible cures to this problem.

To extract the nucleon axial charge, we use a spatial component of the isovector axial current, $A_{k}(x)$. We proceed by taking a ratio of correlation functions,

$$
R\left(t, t_{s}\right)=\frac{C_{3}\left(\overrightarrow{0}, t, t_{s}\right)}{C_{2}\left(\overrightarrow{0}, t_{s}\right)}
$$

where $C_{2}\left(\vec{q}, t_{s}\right), C_{3}\left(\vec{q}, t, t_{s}\right)$ are two- and three-point functions, respectively:

$$
\begin{aligned}
& C_{2}\left(\vec{q}, t_{s}\right)=\sum_{\vec{x}}\left\langle\Gamma_{\alpha^{\prime} \alpha} J_{\alpha}\left(\vec{x}, t_{s}\right) \bar{J}_{\alpha^{\prime}}(0)\right\rangle e^{-i \vec{q} \cdot \vec{x}}, \\
& C_{3}\left(\vec{q}, t, t_{s}\right)=\sum_{\vec{x}, \vec{y}}\left\langle\Gamma_{\alpha^{\prime} \alpha} J_{\alpha}\left(\vec{x}, t_{s}\right) A_{k}(\vec{y}, t) \bar{J}_{\alpha^{\prime}}(0)\right\rangle e^{-i \vec{q} \cdot \vec{y}} .
\end{aligned}
$$

The interpolating operator $\bar{J}_{\alpha}(x)$ is chosen to have the correct quantum numbers in order to produce a nucleon, and $\Gamma_{\alpha^{\prime} \alpha}$ is a projection matrix, which is used to ensure the correct parity of the created nucleon. In the correlation functions, $t_{s}$ denotes the Euclidean time separation between the source and the sink, and $t$ is the Euclidean time separation from the source to the axial current insertion point. From the imaginary part of this ratio, the nucleon axial charge can be extracted as

$$
\operatorname{Im}\left[R\left(t, t_{s}\right)\right] \stackrel{t_{s}, t \rightarrow \infty}{=} g_{\mathrm{A}}^{\text {bare }} .
$$

Taking the contribution from the first excited state to $C_{3}\left(t, t_{s}\right)$ and $C_{2}\left(t_{s}\right)$ into account $[1,2]$, the asymptotic behavior of the ratio can be expressed as

$$
R\left(t, t_{s}\right)=g_{\mathrm{A}}^{\mathrm{bare}}+c_{1} e^{-\Delta t}+c_{2} e^{-\Delta\left(t_{s}-t\right)}+c_{3} e^{-\Delta t_{s}}+\ldots, \quad\left(t_{s}, t \rightarrow \infty\right)
$$

where the energy gap, $\Delta$, is the energy difference between the ground and the first excited state carrying the same quantum numbers as the nucleon at rest. In principle, the energy gap can be extracted from the two-point correlation function; however, we have found the extraction to be difficult with the data available to us. In some of the fits described below, we therefore choose the approximation $\Delta=2 m_{\pi}$.

To test the various methods for extracting the axial charge, we use one ensemble with two dynamical flavours of $\mathrm{O}(a)$ improved Wilson fermions. The most relevant parameters of this ensemble are displayed in Table 1. This ensemble was generated as part of the CLS initiative, in which the deflation-accelerated DD-HMC algorithm [3, 4] was used. A total of 600 measurements were performed by using four sources (equally spaced in the temporal direction) on each of 150 highly decorrelated configurations. Gaussian smearing [5] supplemented by APE-smeared links [6] was used to suppress excited-state contaminations. For some earlier analyses of the nucleon axial charge, as well as the electromagnetic form factors of the nucleon, on the CLS ensembles see $[7,8]$. 


\begin{tabular}{c|c|c|c|c|c|c|c}
\hline Label & volume & $t_{s}$ & $\beta$ & $a[\mathrm{fm}]$ & $a m_{\pi}$ & $N_{\text {cfg }}$ & $N_{\text {src }}$ \\
\hline $\mathrm{N} 4$ & $96 \times 48^{3}$ & $13,16,19,22$ & 5.5 & 0.050 & $0.1358(3)$ & 150 & 4 \\
\hline
\end{tabular}

Table 1: Details of the lattice ensemble used in this calculation, including lattice volume, source-sink separations $t_{s}$ used, coupling constant $\beta$, lattice spacing $a$, pion mass $m_{\pi}$, and numbers of configurations $N_{\mathrm{cfg}}$ and sources $N_{\text {src }}$ used.

\section{Comparing different fitting strategies}

\subsection{Excited-state fits}

The most straightforward way of incorporating the effects of excited states into an extraction of $g_{\mathrm{A}}^{\text {bare }}$ is to fit the measured $R\left(t, t_{s}\right)$ to Eq. (1.5) over a range of different $t$ and $t_{s}$ simultaneously. Because the values of $R\left(t, t_{s}\right)$ at different $t$ and $t_{s}$ are statistically correlated, it is desirable to perform a fully correlated fit to all data. Computing the correlated $\chi^{2}$ requires inverting the covariance matrix $K$ of the data. With a limited number $N$ of samples, it may be unrealistic to estimate the inverse of the full $M \times M$ covariance matrix, where $M$ is the number of different $\left(t, t_{s}\right)$ combinations. In particular for the case that $N$ is not very large, the estimated $\chi^{2}$ may be unreliable as a goodnessof-fit estimator [9].

In our correlated excited-state fits, more than $M=50$ different $\left(t, t_{s}\right)$ combinations were fitted with $N=150$ pre-binned data samples. Since many elements of the covariance matrix are poorly determined, these fits suffer from instabilities and may not describe the central values of the data particularly well (cf. the left panel of Fig. 1 for an example). To avoid these instabilities, we have attempted the following procedure. We diagonalized the covariance matrix and computed the standard deviation of the eigenvalues, which requires considering jackknife samples within each jackknife sample. Each eigenvalue was replaced by the larger of its central value and three times its standard deviation to form a modified matrix $\tilde{K}$, which has the same eigenvectors as $K$ but some of whose eigenvalues are larger. Using $\tilde{K}$ instead of $K$ is a conservative procedure, since $v^{\mathrm{T}} \tilde{K} v \geq v^{\mathrm{T}} K v$ for all $v$, and amounts to increasing the error bar of some linear combinations of the data points by an amount which is formally of order $N^{-1 / 2}$. We expect that $\tilde{K}^{-1}$ should be a more stable estimator of the inverse of the covariance matrix than $K^{-1}$. However, performing the correlated fit with this estimator, we still observe a counterintuitive behavior similar to the one observed in the left panel of Fig. 1. Although this deserves further investigation, it appears that the poor description of the data is not exclusively associated with the smallest eigenvalues.

A pragmatic way to proceed is to perform more physics-motivated approximations to the covariance matrix. As is well-known, the entries of the covariance matrix of primary observables such as $C_{2}$ and $C_{3}$ can be interpreted as correlation functions in the considered ensemble [9]. For the nucleon two-point function, it is easy to see that for large times $t$ and $t^{\prime}$, the off-diagonal elements of $K$ are suppressed by a factor $\exp \left(-\left|t-t^{\prime}\right|\left(2 m_{N}-3 m_{\pi}\right)\right)$. Motivated by this observation, we introduce the "block-correlated fit", which neglects the correlations between data points at different values of $t_{s}$, which we expect to be smaller and less well-determined than those between points at the same value of $t_{s}$. In other words, we approximate the covariance matrix with a block-diagonal matrix by setting its elements $K\left(t, t_{s} ; t^{\prime}, t_{s}^{\prime}\right)$ relating data points with $t_{s} \neq t_{s}^{\prime}$ to zero, while keeping 

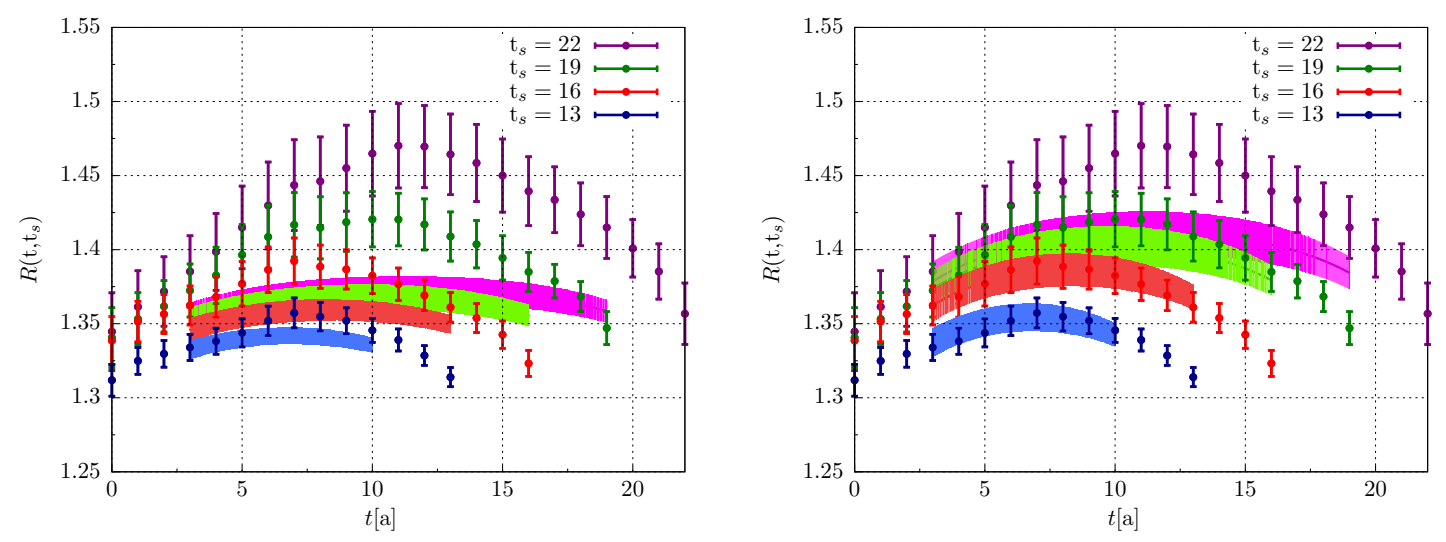

Figure 1: The unrenormalized ratio $R\left(t, t_{s}\right)$ and fits for $t_{s} / a=13,16,19,22$. Left: fully correlated fits. Right: block-correlated fits, where the correlations between points with different $t_{s}$ are neglected.

all other elements unchanged. From the example shown in Fig. 1, it can be seen that this helps to stabilize the fit and to improve the description of the data (cf. also Table 2).

\subsection{The summation method}

An alternative method to extract $g_{\mathrm{A}}^{\text {bare }}$, which avoids the need to perform fits to large number of data points, is the use of summed operator insertions, for which the leading asymptotic approximation is given by [10]

$$
S\left(t_{s}\right)=\sum_{t=t_{\mathrm{cut}}}^{t_{s}-t_{\mathrm{cut}}} R\left(t, t_{s}\right) \stackrel{t_{s} \rightarrow \infty}{\longrightarrow} g_{\mathrm{A}}^{\mathrm{bare}} x+C, \quad x\left(t_{s}\right)=t_{s}+1-2 t_{\mathrm{cut}}
$$

We have allowed for the possibility of reducing the effect of higher excited states by dropping the data points with a distance of less than $t_{\text {cut }}$ from the source or sink in a symmetrical fashion, allowing us to determine $g_{\mathrm{A}}^{\text {bare }}$, as well as the excited-state parameter $C$, from a simple straightline fit. In the simplest version of this method, the $\mathrm{O}\left(e^{-\Delta t_{s}}\right)$ contribution is ignored. A more sophisticated version of the summation method is obtained if we rewrite the summed ratio without neglecting the $\mathrm{O}\left(e^{-\Delta t_{s}}\right)$ contributions,

$$
S\left(t_{s}\right)=x\left(g_{\mathrm{A}}^{\mathrm{bare}}+B e^{-\Delta x}\right)+C\left(1-e^{-\Delta x}\right),
$$

where an additional parameter $B$ has to be determined. Fig. 2 shows a comparison between the fits obtained using Eq. (2.1) ("standard summation method") and those including the excited state contribution in Eq. (2.2) ("extended summation method"). It can be seen that the slope obtained from the standard summation method is dominated by the data points at the smallest values of $t_{s}$, which have the smallest statistical errors, whereas the extended summation method is able to describe the steeper slope suggested by the (statistically less precise) data points at the largest values of $t_{s}$ (see also Table 2). 

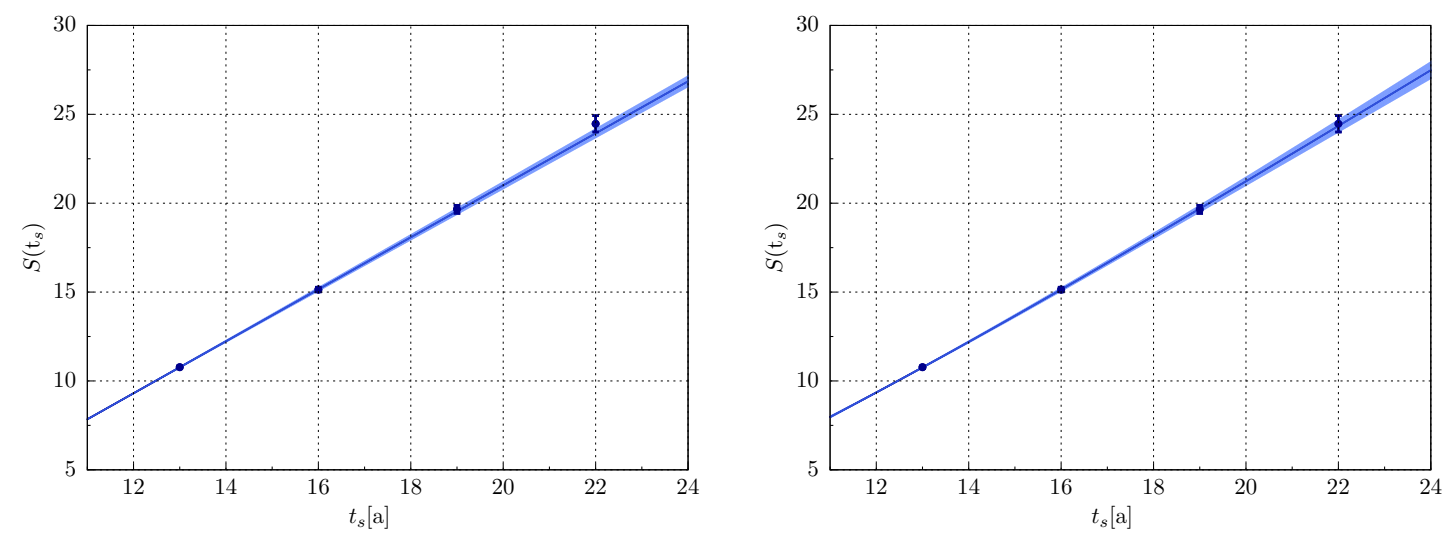

Figure 2: Summation method; the bare axial charge is given by the slope. Left: standard summation method; the summed ratios are fitted with Eq. (2.1) as a straight line. Right: extended summation method; the fit is to Eq. (2.2) with higher-order excited-state contributions included.

\subsection{The midpoint method}

For $0 \ll t \ll t_{s}$, the term of $\mathrm{O}\left(e^{-\Delta t_{s}}\right)$ in Eq. (1.5) is parametrically small relative to the other corrections. To minimize the $t$-dependent corrections, we can consider the point at $t=t_{s} / 2$, for which both are $\mathrm{O}\left(e^{-\Delta_{\frac{5}{5}}}\right)$. We assume that in the extraction of $g_{\mathrm{A}}^{\text {bare }}$ the excited state coefficients $c_{1}$ and $c_{2}$ in Eq. (1.5) are approximately identical, as suggested by the symmetric shape of the data. It is therefore an acceptable approximation to consider only the data point $M\left(t_{s}\right)=R\left(t=\frac{t_{s}}{2}, t_{s}\right)$, or $M\left(t_{s}\right)=R\left(t=\frac{t_{s} \pm 1}{2}, t_{s}\right)$ if $t_{s}$ is odd ("midpoint method"), and parameterize it by combining these two contributions

$$
M\left(t_{s}\right)=g_{\mathrm{A}}^{\mathrm{bare}}+A x\left(t_{s}\right), \quad x\left(t_{s}\right)= \begin{cases}e^{-\Delta \frac{t_{s}}{2}} \cosh \left(\frac{\Delta}{2}\right) & t_{s} \text { is odd } \\ e^{-\Delta \frac{t_{s}}{2}} & t_{s} \text { is even }\end{cases}
$$

This method then allows us to extract $g_{\mathrm{A}}^{\text {bare }}$ as the $y$-axis intercept in a simple straight-line fit to $M\left(t_{s}\right)$ as a function of $x\left(t_{s}\right)$. To allow for a comparison between this fit and the standard summation method, we rewrite the summed ratio by dividing both sides of Eq. (2.1) by $x=t_{s}+1-2 t_{\text {cut }}$,

$$
\frac{S\left(t_{s}\right)}{x}=g_{\mathrm{A}}^{\mathrm{bare}}+\frac{C}{x},
$$

and present the fit results in Table 2 and Fig. 3.

\section{Conclusions and outlook}

As each of the fitting strategies introduced in the preceding section has its own systematic errors, we attempt to obtain a handle on the systematic errors arising from excited state contributions by extracting $g_{\mathrm{A}}^{\text {bare }}$ using each of the fit strategies and and comparing the results, which are listed in Table 2. The value of $g_{\mathrm{A}}$ given in the table has been renormalized with the renormalization constant $Z_{\mathrm{A}}=0.8007(96)$ from [11].

From the table we see that several of the different strategies differ by more than their respective statistical errors, indicating that there may be some residual systematic errors from excited-state 

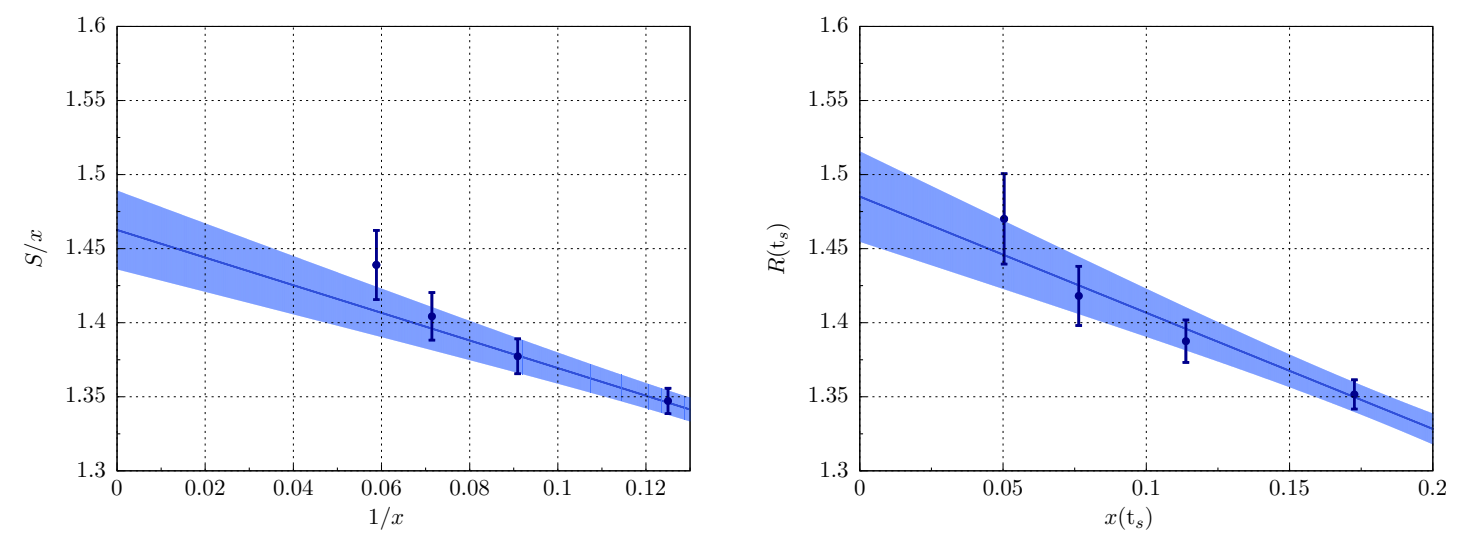

Figure 3: Comparison of summation and "midpoint" methods; the axial charge is given by the intercept. Left: an alternative way to represent the standard summation method using Eq. (2.4). Right: the "midpoints" fit of Eq. (2.3), where at each $t_{s}$ only the data point at $t=t_{s} / 2$ is considered.

\begin{tabular}{|c|c|c|c|c|}
\hline Fitting method & $N_{\mathrm{par}}$ & $g_{\mathrm{A}}$ & excited contamination & $\chi_{\text {red }}^{2}$ \\
\hline $\begin{array}{l}\text { fully correlated excited-state fit } \\
\text { Fig. } 1 \text { left, Eq. (1.5) }\end{array}$ & $1+3$ & $1.097(6)$ & $\begin{array}{c}c_{1}=-0.041(12) \\
c_{2}=-0.034(6) \\
c_{3}=-0.595(185)\end{array}$ & 2.247 \\
\hline $\begin{array}{l}\text { "block correlated" excited-state fit } \\
\text { Fig. } 1 \text { right, Eq. (1.5) }\end{array}$ & $1+3$ & $1.140(13)$ & $\begin{array}{c}c_{1}=-0.074(18) \\
c_{2}=-0.064(13) \\
c_{3}=-1.090(325)\end{array}$ & 1.385 \\
\hline $\begin{array}{l}\text { summation (standard) } \\
\text { Fig. } 2 \text { left, Eq. (2.1) }\end{array}$ & $1+1$ & $1.171(21)$ & $C=-0.747(148)$ & 0.076 \\
\hline $\begin{array}{l}\text { summation (extended) } \\
\text { Fig. } 2 \text { right, Eq. (2.2) }\end{array}$ & $1+2$ & $1.275(69)$ & $\begin{array}{c}B=2.5(2.0) \\
C=-5.2(2.2)\end{array}$ & 0.017 \\
\hline $\begin{array}{c}\text { midpoint } \\
\text { Fig. } 3 \text { right, Eq. (2.3) }\end{array}$ & $1+1$ & $1.189(24)$ & $A=-0.314(64)$ & 1.150 \\
\hline
\end{tabular}

Table 2: Comparison of all methods for the extraction of $g_{\mathrm{A}} . N_{\mathrm{par}}$ is the number of fit parameters $\left(g_{\mathrm{A}}\right.$ and the excited-state contaminations listed in the third column).

contaminations. The range of independent methods at our disposal suggests that we could perform the same range of analyses on a set of lattice ensembles and combine each of the fitting strategies with a range of chiral and continuum extrapolations to estimate the systematic error using a pseudobootstrap method [12].

We have studied a range of different fitting strategies to extract the axial charge of the nucleon from ratios of correlation functions. While fully correlated fits for the nucleon axial charge tend to become unstable, a "block-correlated fit" can help stabilize the simultaneous excited state fits, while taking into account the dominant correlations in the data. The "midpoint" method provides a viable alternative to the standard summation method, with the advantage that the required extrapolation is much shorter (cf. Fig. 3). Therefore the result of the extrapolation is almost identical to the data point at the largest $t_{s}$, rendering the result much less sensitive to the point at the smallest $t_{s}$. On the 
other hand, the "standard summation method", has the advantage of not requiring any particular assumption about the value of the energy gap $\Delta$; the contribution proportional to $B$ in Eq. (2.2) only needs to be small enough to neglect.

Further investigations using other ensembles with different lattice spacings and pion masses, and different sets of source-sink separations, are necessary for a more complete picture. Furthermore, ensembles with increased statistics can provide more reliable data that should allow for more stable fits with a more accurately known covariance matrix.

\section{Acknowledgments}

Our calculations were performed on the "Wilson" HPC Cluster at the Institute for Nuclear Physics, University of Mainz. We are grateful to Dalibor Djukanovic and Christian Seiwerth for technical support. This work was supported by DFG (SFB 1044 and grant HA4470/3-1) and the Rhineland-Palatinate Research Initiative. We are grateful to our colleagues within the CLS initiative for sharing ensembles.

\section{References}

[1] J. Green, S. Krieg, J. Negele, A. Pochinsky, and S. Syritsyn, PoS(LATTICE2011) 157 (2011) [arXiv:1111.0255].

[2] S. Capitani, B. Knippschild, M. Della Morte and H. Wittig, PoS(LATTICE2010) 147 (2010) [arXiv:1011.1358].

[3] M. Lüscher, JHEP 12011 (2007) [arXiv: 0710.5417 ].

[4] M. Lüscher, Comput. Phys. Commum. 165199 (2005) [hep-lat / 0409106 ].

[5] S. Güsken, et al., Phys. Lett. B 227266 (1989).

[6] M. Albanese et al. [APE Collaboration], Phys. Lett. B 192 (1987) 163.

[7] S. Capitani, et al., Phys. Rev. D 86 (2012) 074502 [arXiv: 1205 . 0180].

[8] S. Capitani, et al., PoS(LATTICE2012) 177 (2012) [arXiv:1211.1282].

[9] C. Michael and A. McKerrell, Phys. Rev. D 51 (1995) 3745 [hep-lat/9412087];

C. Michael, Phys. Rev. D 49 (1994) 2616 [hep-lat/9310026].

[10] L. Maiani, G. Martinelli, M. L. Paciello, and B. Taglienti, Nucl. Phys. B 293420 (1987).

[11] M. Della Morte, R. Sommer, and S. Takeda, Phys. Lett. B 672407 (2009) [arXiv: 0807. 1120].

[12] S. Dürr, Z. Fodor, J. Frison, C. Hoelbling, R. Hoffmann, S. D. Katz, S. Krieg and T. Kurth et al., Science 322 (2008) 1224 [arXiv:0906.3599 [hep-lat]]. 